Research Article

\title{
Imaging Algorithm-Based Real-Time Shear Wave Elastography Combined with Thyroglobulin Antibodies in Diagnosis of Thyroid Cancer
}

\author{
Xinjing Xie $\mathbb{D}^{1},{ }^{1}$ Jun Yang $\mathbb{D}^{2},{ }^{2}$ Guoxin Liang $\mathbb{D}^{1},{ }^{1}$ Chunxiang $\mathrm{Li}\left(\mathbb{D},{ }^{3}\right.$ and Zhongyuan Li $\mathbb{D}^{4}$ \\ ${ }^{1}$ Department of Ultrasonography, The First Affiliate Hospital of Qiqihar Medical College, Qiqihar 161041, Heilongjiang, China \\ ${ }^{2}$ Department of Price Division, The First Affiliate Hospital of Qiqihar Medical College, Qiqihar 161041, Heilongjiang, China \\ ${ }^{3}$ Department of Nuclear Medicine, The First Affiliate Hospital of Qiqihar Medical College, Qiqihar 161041, Heilongjiang, China \\ ${ }^{4}$ Department of Nuclear Medicine Department, The First Affiliate Hospital of Qiqihar Medical College, Qiqihar 161041, \\ Heilongjiang, China
}

Correspondence should be addressed to Zhongyuan Li; whp6841635@qmu.edu.cn

Received 11 May 2021; Revised 19 June 2021; Accepted 8 July 2021; Published 26 July 2021

Academic Editor: Gustavo Ramirez

Copyright (C) 2021 Xinjing Xie et al. This is an open access article distributed under the Creative Commons Attribution License, which permits unrestricted use, distribution, and reproduction in any medium, provided the original work is properly cited.

\begin{abstract}
To analyze the effect of real-time shear wave elastography (SWE) optimized by mathematical algorithms combined with thyroglobulin antibodies (TGAb) on the clinical diagnosis of differentiated thyroid carcinoma (DTC), a hybrid displacement estimation algorithm based on weighted phase separation and two-dimensional cross correlation was proposed. 102 patients with DTC were divided into a test group (TGAb-positive) and a control group (TGAb-negative). Real-time SWE based on hybrid displacement estimation algorithm was performed. Receiver operating characteristic (ROC) curve was adopted to analyze the characteristics of real-time SWE and its combination with TGAb to detect the sensitivity, specificity, and area under the curve (AUC) of the malignant degree of thyroid cancer. The results showed that the preoperative thyroid-stimulating hormone (TSH), thyroglobulin autoantibodies (TGAb), and thyroid peroxidase antibody (TPOAb) of TGAb-positive patients were higher than those of the TGAb-negative group $(P<0.05)$. The preoperative tumors of TGAb-positive patients were multifocal, and the tumor size was larger than that of the TGAb-negative patients $(P<0.05)$. The maximum Young's modulus $E_{\max }$ of TGAb-positive patients was greater than that of TGAb-negative group $(P<0.05)$. In addition, there was a very significant positive correlation between the patient's TGAb level and $E_{\max }$ of Young's modulus $(P<0.001)$. The sensitivity, specificity, and AUC of the joint detection of real-time SWE $E_{\max }$ and TGAb for the malignant degree of thyroid cancer were significantly greater than those of the single real-time SWE and TGAb, and the difference was substantial $(P<0.05)$. In short, joint detection of real-time SWE based on hybrid displacement estimation algorithm combined with TGAb had high sensitivity, specificity, and AUC for the diagnosis of DTC, which was suitable for clinical application.
\end{abstract}

\section{Introduction}

Differentiated thyroid carcinoma (DTC) is one of the common endocrine malignancies. In recent years, its incidence has shown an obvious upward trend $[1,2]$. Clinically, the diagnosis of DTC has a high rate of misdiagnosis due to the largest diameter of the tumor, the distribution of special subtypes, atypical clinical symptoms, and small lesions. Although the progress of papillary thyroid carcinoma (PTC) is slow and the biological behavior tends to be benign, it can spread in the glands and metastasize to regional lymph nodes, leading to further lesions, so preoperative diagnosis is very important. At present, advanced and effective imaging techniques are used for inspection to improve the diagnosis rate of DTC [3]. In recent years, shear wave elastography (SWE), which is implemented based on conventional ultrasound examination, has gradually become the preferred method for thyroid disease. It can detect tiny nodules about 1 to $2 \mathrm{~mm}$ and clearly shows the gravel-like calcification in the tiny nodules and the changes in the nature of the 
surrounding lymph nodes [4]. Due to the limitation of image clarity in conventional ultrasound images, the imaging effect of thyroid nodules is not good, which is not easy to distinguish from other normal tissues, resulting in missed diagnosis. Therefore, improving the corresponding quality indicators of ultrasound images is of great significance for the early diagnosis of patients with thyroid cancer.

As a new technology in recent years, SWE uses ultrahighspeed imaging technology to generate various forms of sound waves through a multiwave imaging platform and a probe chip. It adopts acoustic radiation pulse control technology, continuous focusing at various depths of the tissue, and forming a "Mach cone" phenomenon to promote the generation of shear waves [5]. Since SWE has a quality mode to control the quality of the elastic image during the inspection process, it has good objectivity and strong repeatability. It can display the diseased tissue in time and does not require artificial pressure on the patient's tissue, which can reduce the impact on the patient. Therefore, it has become an important auxiliary diagnosis for the differential diagnosis of benign and malignant thyroid nodules [6]. Therefore, it has become an important auxiliary diagnosis for the differential diagnosis of benign and malignant thyroid nodules. Real-time SWE can visually display the color coding of elastic images under the real-time guidance of twodimensional images and directly measures the absolute value of Young's modulus of thyroid nodules, which is expressed quantitatively in the form of Young's value (kPa). SWE uses color images to intuitively reflect the elastic distribution of the organization in real time. SWE can measure many Young's moduli, including the maximum value $\left(E_{\max }\right)$, average value $\left(E_{\text {mean }}\right)$, minimum value $\left(E_{\min }\right)$, and standard deviation $\left(E_{\mathrm{sd}}\right)$ of the measured structure. It can comprehensively and accurately quantitatively analyze the hardness of the lesion tissue and the changes with the surrounding tissues. Moreover, it can well describe the morphological characteristics of the lesion and can clarify the state of the lesion, reflecting the characteristics of the blood supply of the tumor and the relationship with the surrounding tissue structure. It can also objectively display the size and structure of the tumor. Unfortunately, due to the noise and artifacts in SWE imaging, there is a certain degree of missed diagnosis rate in clinical diagnosis, and other methods are needed for auxiliary diagnosis [2].

Thyroglobulin antibodies (TGAb) are an emerging diagnostic marker for thyroid cancer tumors which have emerged in recent years. TGAb is the corresponding antibody of thyroglobulin $(\mathrm{Tg})$. As one of the main serum tumor markers in DTC patients, Tg can monitor the tumor recurrence and metastasis after treatment. High titer TGAb will affect the accuracy of $\mathrm{Tg}$ determination and reduces the sensitivity of $\mathrm{Tg}$ as a tumor marker [7-9]. Some studies suggested that TGAb alone can also be used as a potential predictor of DTC, which has become a DTC substitute tumor marker. TGAb is used to assist physicians in the effective diagnosis of thyroid cancer to improve the accuracy of DTC diagnosis $[10,11]$. Therefore, combination of realtime SWE and TGAb under the imaging algorithm was used to explore the rapid clinical diagnosis of thyroid cancer.
In summary, a hybrid displacement estimation algorithm with weighted phase separation and two-dimensional cross-correlation was proposed in the traditional imaging algorithm design, which was applied to the image analysis of 102 patients with DTC in real-time SWE. The optimization effect of the algorithm was evaluated through indicators such as sensitivity, specificity, and AUC. Receiver operating characteristic (ROC) curve, Young's value $E_{\max }$, etc. were used to evaluate the diagnosis performance for thyroid cancer, so as to comprehensively evaluate the application value of real-time SWE under the imaging algorithm combined with TGAb in the diagnosis of DTC.

\section{Materials and Methods}

2.1. Research Objects. 102 patients with DTC who were pathologically confirmed by pathology in the hospital were selected as the research objects and were divided into a test group (TGAb-positive) and a control group (TGAb-negative). Among them, 59 were males and 45 were females, aged 29-65 years. The patients were randomly divided into test group (TGAb positive) and control group (TGAb negative), with 51 people in each group. In the experimental group (TGAb-positive), TGAb $\geq 115 \mathrm{IU} / \mathrm{mL}$, there were a total of 56 patients, TGAb level was 498.0 (356.5-998.6) IU/mL, and average age was $41.74 \pm 11.87$ years, of which 15 were males and 36 were females. In the control group (TGAb-negative), $\mathrm{TGAb}<115 \mathrm{IU} / \mathrm{mL}$, TGAb level was 9.78 (8.79-12.96) $\mathrm{IU} / \mathrm{mL}$, and average age was $39.67 \pm 9.88$ years, including 21 males and 30 females. The study had been approved by the ethics committee of the hospital, and the patients and their families included in the study knew the study process and signed the informed consent.

Inclusion criteria were as follows: (i) patients without history of thyroid surgery before the experiment, (ii) patients with complete basic clinical data, and (iii) patients with preoperative serological examination results. Exclusion criteria were as follows: (i) patients whose surgery was performed in a different hospital or whose surgical method was partial thyroidectomy or lymph node dissection, (ii) patients with incomplete pathological data, and (iii) patients who had not been tested for serological indicators or had incomplete inspection indicators.

2.2. SWE Image Acquisition. The Mindray Resona 7 ultrasonic color Doppler diagnostic apparatus equipped with SWE software was used, the probe model was L11-3U, and the frequency was 5.6, 10.0 MHz. The patient took a supine position and breathed calmly. The thyroid imaging mode was set to perform routine ultrasound examination of thyroid nodules, such as location, size, number, blood flow, cystic change, and calcification. The largest longitudinal section of the thyroid nodule was taken. After waiting for $3-5$ seconds, the image was frozen after the image was stable. The quality mode of SWE was observed, and it was switched to the measurement mode after the credibility index was as close as possible to $100 \%$ and the lesion area was uniformly green. If the reliability index was low and there was a lot of 
yellow in the lesion area, the measurement was not accurate and needed to be measured again. For the area of interest, the measurement method that comes with the ultrasonic instrument was adopted for further measurement. During the measurement, the liquid anechoic area and the large calcification should be avoided as much as possible, and the lesion should be covered as much as possible. Three repeated operations were performed for each lesion. The measurement data of the measurement lesion should include the maximum value $\left(E_{\max }\right)$, average value $\left(E_{\text {mean }}\right)$, and minimum value $\left(E_{\text {min }}\right)$ of the elastic modulus value. Statistics of routine indicators such as tumor size and number of lesions of patients were conducted, and all operations were performed by physicians who had received SWE examination technology training.

\subsection{Real-Time Shear Wave Model Construction Based on} Imaging Algorithm. Imaging algorithm was employed to optimize real-time SWE, which was of great application value in clinical tumor diagnosis, and can greatly reduce the degree of dependence on the subjective judgment of physicians. The amplitude modulation correction (AMC) can reduce the amplitude modulation noise in the traditional calculation algorithm structure, which also estimated the displacement through the fluctuation of the signal amplitude. Its computing efficiency was very high, and its performance was superior to stretching technology. The strain estimation based on the amplitude modulation theory was expressed by the phase difference as follows:

$$
\varepsilon=\frac{a_{2}-a_{1}}{N_{2}-N_{1}}
$$

In equation (1), $a_{2}$ and $a_{1}$ represented the displacement of the continuous window, and $N_{2}$ and $N_{1}$ represented the relative position of the displacement.

First, the signal model was set as follows:

$$
\begin{gathered}
b_{1}(q)=b_{c 1}(q)+g b_{j 1}(q), \\
b_{2}(q+d(q))=b_{c 2}(q+d(q))+g b_{j 2}(q+d(q)) .
\end{gathered}
$$

In equations (2) and (3), $b_{1}(q)$ and $b_{2}(q)$ were the analytical signals of presignal and postsignal, respectively, which were obtained through Hilbert transform or IQ modulation.

Then, the AMC technique was used in the phase estimation, which was expressed as follows:

$$
\widehat{h}_{m} \approx \frac{\sum_{q=m \Delta q}^{m \Delta q+Q} V(q) d(q)}{\sum_{q=m \Delta q}^{m \Delta q+Q} V(q)} .
$$

In equation (4), $q$ was the axial sampling point, and $q=0$ was the surface of the transducer. $\Delta q$ was the sampling frequency, $Q$ was the window length, and $d(q)$ was the real displacement at $q$. The position of $\widehat{h}_{m}$ was related to the weight, such as amplitude modulation. The weight can not only be the amplitude, but all signal attributes can be used as the weight. $d(q)$ was expressed as

$$
d(q) \approx \alpha+z q
$$

In equation (5), $z$ was the tissue strain, and the position estimate $\tau_{m}$ at $\widehat{h}_{m}$ was defined as follows:

$$
\widehat{\tau}_{m}=\frac{\sum_{q=m \Delta q}^{m \Delta q+Q} V(q) q}{\sum_{q=m \Delta q}^{m \Delta q+Q} V(q)} .
$$

Displacement estimation based on phase information was as follows. The phase method processed the analytic signal and obtained the analytic signal through the Hilbert transform. The complex cross correlation was expressed as follows:

$$
\left\langle k_{1}, k_{2}\right\rangle\left(m \Delta q, \tilde{d}_{x}\right)=\sum_{q=m \Delta q}^{m \Delta q+Q} k_{1}^{*}(q) q_{2}\left(q+\tilde{d}_{x}\right) .
$$

In equation (7), $k_{1}$ and $k_{2}$ were the analytic signals before and after compression, $k_{1}^{*}$ was the conjugate complex number of $k_{1}, m \Delta q$ was the start of the signal of the $m$ th window, $Q$ was the window length, and $X$ was the estimated displacement value. The phase expression was as follows:

$$
\Phi\left(m \Delta q, \tilde{d}_{x}\right)=\angle\left\langle k_{1}, k_{2}\right\rangle\left(m \Delta q, \widetilde{d}_{x}\right) .
$$

Weight phase separation (WPS) was adopted for precise estimation of axial displacement. WPS was set as the matching criterion to obtain the subsampling point-level axial displacement accuracy. The following equation was used for iteration:

$$
\tilde{d}_{x+1}=\tilde{d}_{x}+\frac{\sum_{(i, j) \in Q} U(i, j)\left[\varphi_{\text {pre }}(i, j)-\varphi_{\text {post }}\left(i+d_{i}, j+d_{j}\right)\right]}{u_{0} \sum_{(i, j) \in Q} U(i, j)} .
$$

In equation (9), $\tilde{d}_{x}$ and $\tilde{d}_{x+1}$ were the displacements, $\varphi_{\text {pre }}$ and $\varphi_{\text {post }}$ were the phase of the RF signal before and after compression, and $u_{0}$ was the center frequency. $U(i, j)$ was the weight, and its expression was as follows:

$$
U(i, j)=\sum_{(i, j) \in Q}\left[q_{1}(i, j)+q_{1}\left(i+d_{i}, j+d_{j}\right)\right] .
$$

In equation (10), $q_{1}$ and $q_{2}$ were the amplitude signals corresponding to presignal and postsignal. In this way, the algorithm was divided into two levels. The first-level displacement estimation was rough estimation, which was accurate to the sampling point, and the second displacement estimation was the net estimation, which improved the axial resolution. Moreover, the displacement data was smoothed. In the strain estimation, to avoid the gradient calculation to further amplify the displacement estimation noise, the leastsquares strain estimation method was adopted.

2.4. Real-Time Shear Wave Elastic Imaging Feature Acquisition and Label Establishment. After processing of the realtime SWE image with a hybrid displacement estimation algorithm based on weighted phase separation and two- 
dimensional cross-correlation, the specific process and effect changes are shown in Figures 1 and 2.

The collected SWE images of 102 patients were optimized by algorithm step by step. Before and after the algorithm processing during the collection optimization period, the processing algorithms included one-dimensional elastic algorithm, two-dimensional elastic algorithm, and two-dimensional cross-correlation hybrid algorithm. The SWE image quality processed by different algorithms was compared and analyzed, and the SWE image optimization effects of each algorithm were summarized.

2.5. The Tumor Pathological Characteristics and Preoperative Serological Index Detection of the Two Groups of Patients. Preoperative serological indexes (thyroid-stimulating hormone (TSH) and thyroid peroxidase antibody (TPOAb)) and pathological characteristics of tumors were compared between the test group and the control group. The following pathological features of the surgically resected specimen were evaluated by at least two experienced pathologists: the size of the tumor (the maximum diameter of the largest tumor was measured) and whether there was peripheral invasion, bilateral, multifocal $(\geq 2$ tumor lesions in one thyroid lobe), lymph node metastasis rate (number of lymph node metastases/number of lymph nodes surgically dissected), lymph node staging (N0: no regional lymph node metastasis; N1A: lymph node metastasis in VI group, namely, pretracheal, paratracheal, and prelaryngeal lymph nodes; N1B: unilateral, bilateral, or contralateral cervical or superior mediastinal lymph node metastases).

2.6. Statistical Methods. The data processing of this study was performed by SPSS 19.0. Counting data was described by percentage $(\%)$, and measurement data was represented by mean \pm standard deviation $(\mathrm{x} \pm s)$. The Mann-Whitney $U$ test method was used to compare the differences in the macroscopic characteristics and imaging omics characteristics of the two groups of patients. The ROC was used to calculate the area under the curve (AUC) and sensitivity, specificity, and accuracy of each feature. $P<0.05$ indicated that the difference was statistically considerable. The $t$-test was used to compare the difference between the two groups for continuous variables that met the normal distribution and the homogeneity of variance. Continuous variables that did not meet the normal distribution (preoperative TSH, TGAb, TPOAb, tumor size, and lymph node metastasis rate) were tested by the rank-sum test (Kruskal-Wallis). Categorical variables (tumor size and multifocality) were tested by chi-square test to compare their constituent ratios between the two groups. $P<0.05$ indicated that the difference was considerable.

\section{Results}

3.1. Quality Evaluation of Algorithm Optimization. Figure 3 shows a comparison diagram of SWE effects under different algorithm processing. Figures 3(a)-3(d) show SWE original image, one-dimensional elastic algorithm processed image, two-dimensional elastic algorithm processed image, and two-dimensional cross-correlation hybrid algorithm processed image, respectively. The image quality of the SWE image processed by the algorithm was improved, and the image improvement effect of the SWE image processed by the two-dimensional cross-correlation hybrid algorithm was the most obvious, followed by the two-dimensional elastic algorithm and the one-dimensional elastic algorithm.

Figure 4 shows the comparison of signal-to-noise ratios of SWE strain diagrams processed by different algorithms. Compared with the one-dimensional elastic algorithm and the two-dimensional elastic algorithm, the two-dimensional cross-correlation hybrid algorithm had better signal-tonoise optimization effect. The signal-to-noise ratio curves of the two-dimensional elastic algorithm and the two-dimensional cross-correlation hybrid algorithm crossed, but the overall SNR optimization effect of the two-dimensional cross-correlation hybrid algorithm was better. Figure 5 showed the comparison of operation time of different algorithms. The calculation time of the two-dimensional cross-correlation hybrid algorithm was longer than that of the two-dimensional elastic algorithm in the early stage, and the calculation time was shorter than that of the two-dimensional elastic algorithm in the later stage. The computing times of the two-dimensional cross-correlation hybrid algorithm and the two-dimensional elastic algorithm were significantly shorter than that of the one-dimensional elastic algorithm.

3.2. Analysis of Preoperative Serum Indexes of Two Groups of Patients. Through the comparisons of the levels of TSH, TGAb, and TPOAb in the preoperative serum indexes of the two groups of patients, it was found that the preoperative TSH, TGAb, and TPOAb of TGAb-positive patients were all greater than those of the TGAb-negative group $(P<0.05)$, as illustrated in Figure 6.

3.3. Comparison of Tumor Pathological Characteristics between the Two Groups. It was found that there were remarkable differences in the tumor size and the number of lesions between the two groups after the tumor pathological characteristics of the two groups of patients were compared. The preoperative tumors of experimental group were multifocal, and the tumor size was larger than that of control group $(P<0.05)$, with considerable differences (Figures 7 and 8$)$.

3.4. Comparison of $E_{\text {max }}$ Value between Two Groups of Patients and Analysis of the Correlation between $E_{\text {max }}$ Value and TGAb Level. Young's modulus $E_{\max }, E_{\text {mean }}$ value, and $E_{\min }$ value of the two groups of patients were measured and compared, and the results showed that maximum Young's modulus $E_{\text {max }}$ of experimental group was superior to that of control group $(P<0.05)$ (Figure 9$)$. Moreover, the patient's TGAb level and the corresponding data of maximum Young's modulus $E_{\max }$ were analyzed, and it was found that there was 


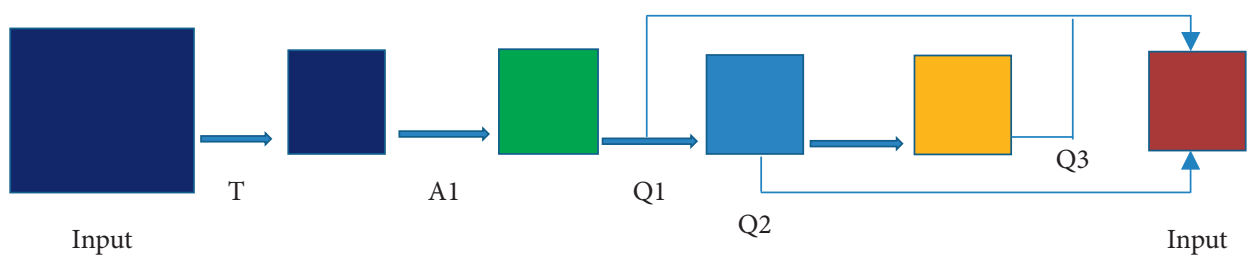

FIGURE 1: Algorithm optimization flowchart (T indicated image compression; A1 indicated displacement estimation plus strain estimation; Q1 indicated phase separation processing; Q2 indicated weighted phase separation processing; Q3 indicated two-dimensional crosscorrelation mixing processing).

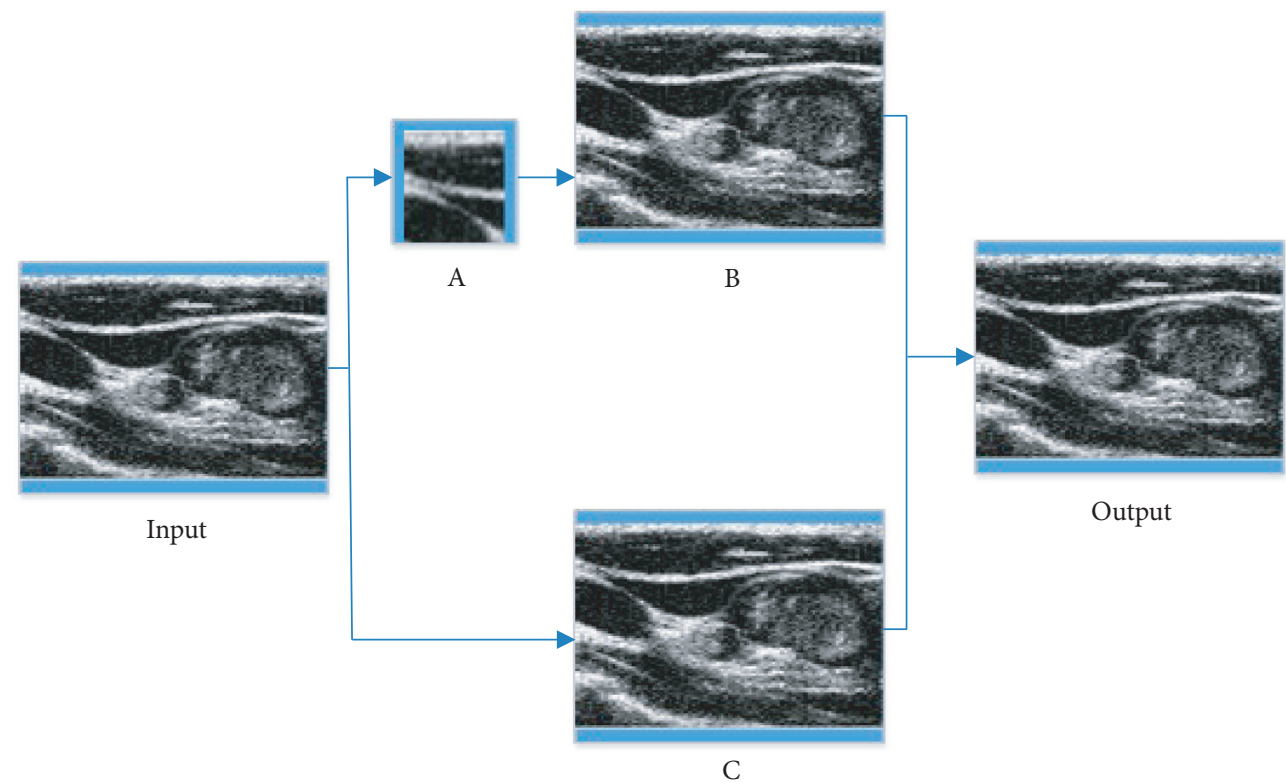

FIGURE 2: Change of algorithm processing effect (A represented the effect after phase separation processing; B represented weighted phase separation processing; $\mathrm{C}$ represented two-dimensional cross-correlation mixing processing).

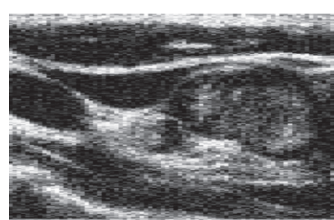

(a)

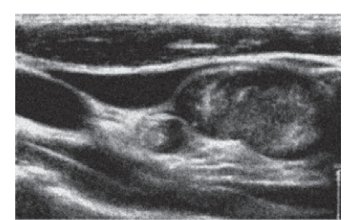

(b)

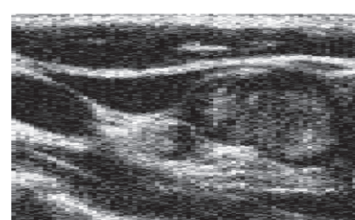

(c)

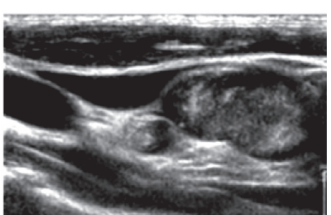

(d)

FIGURE 3: Comparison of SWE effects under a different algorithm processing. (a) Before processing; (b) one-dimensional elastic algorithm processing; (c) two-dimensional elastic algorithm processing; (d) two-dimensional cross-correlation hybrid algorithm processing.

a very significant positive correlation between the two $(P<0.001)$, which indicated that the level of TGAb in the patient's body greatly affected the value of $E_{\max }$, as presented in Figure 10.

\subsection{SWE Image Quality Analysis Based on Imaging Algorithm.} Patient's SWE image optimized by the elastography algorithm was analyzed, and the optimization effect of the algorithm was evaluated regarding the detection sensitivity, specificity, and AUC. Figures 11 and 12 show that the sensitivity, specificity, and AUC of the joint detection of patient real-time SWE $E_{\max }$ and TGAb for the malignant degree of thyroid cancer were dramatically greater than those of a single real-time SWE and TGAb, with statistical significance $(P<0.05)$.

\section{Discussion}

Thyroid cancer is a malignant tumor with a very high incidence. Due to the complex anatomical structure of the human thyroid gland, which is closely connected with multiple organs, and the fact that thyroid cancer is highly erosive, it is easy to invade the surrounding tissue structure 


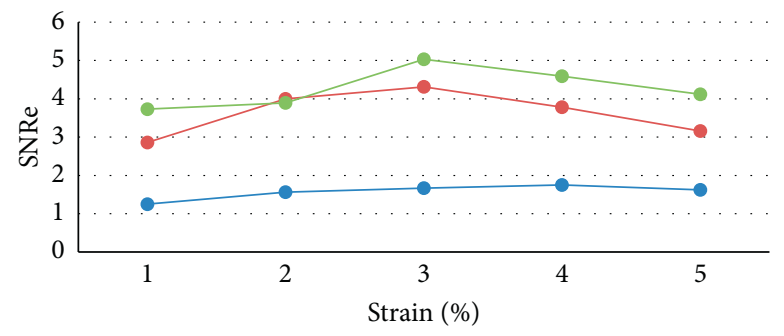

- One-dimensional algorithm

$-\bullet$ Two-dimensional algorithm

$\rightarrow$ Hybrid algorithm

FIgURE 4: Signal-to-noise ratios of SWE strain map processed by different algorithms.

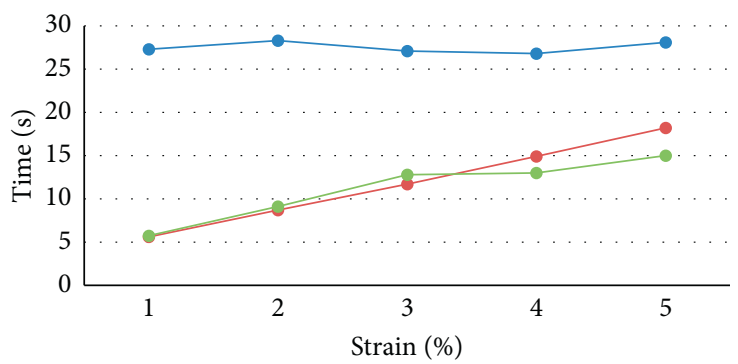

- One-dimensional algorithm
$\multimap-$ Two-dimensional algorithm
$\multimap-$ Hybrid algorithm

Figure 5: Comparison of operation time of different algorithms.

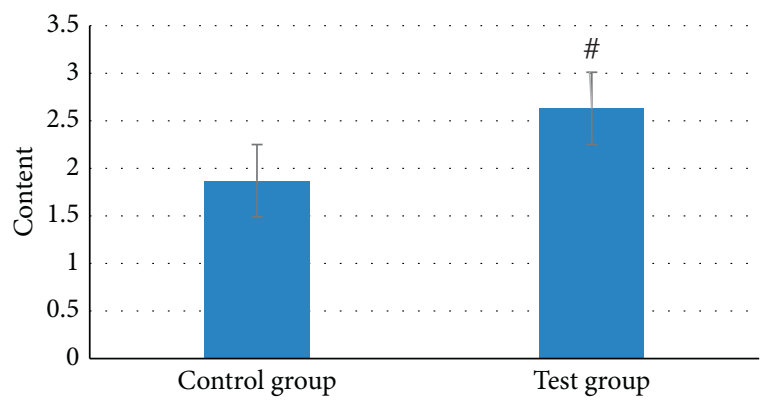

(a)

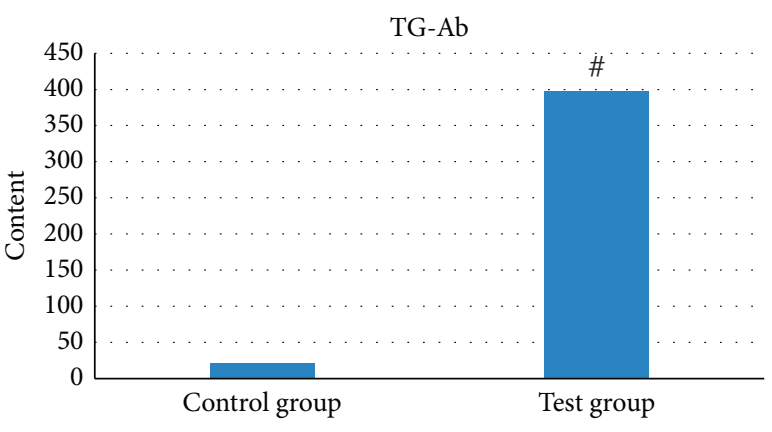

(b)

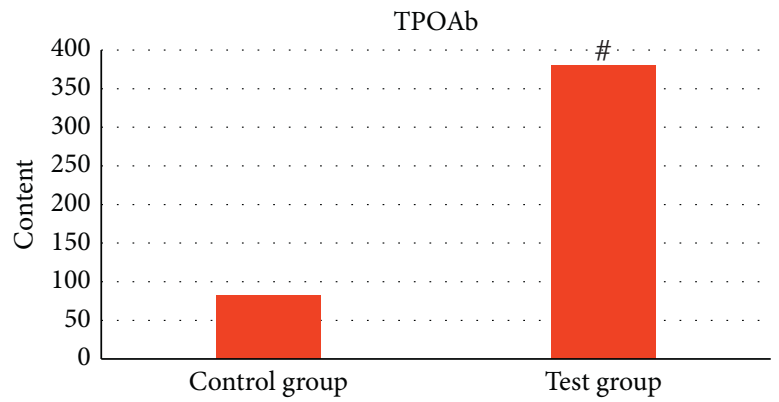

(c)

FIGURE 6: (a) Comparison of serum TSH levels in the two groups of patients before surgery. (b) Comparison of serum TGAb levels in the two groups of patients before surgery. (c) Comparison of serum TPOAb levels in the two groups of patients before surgery. 


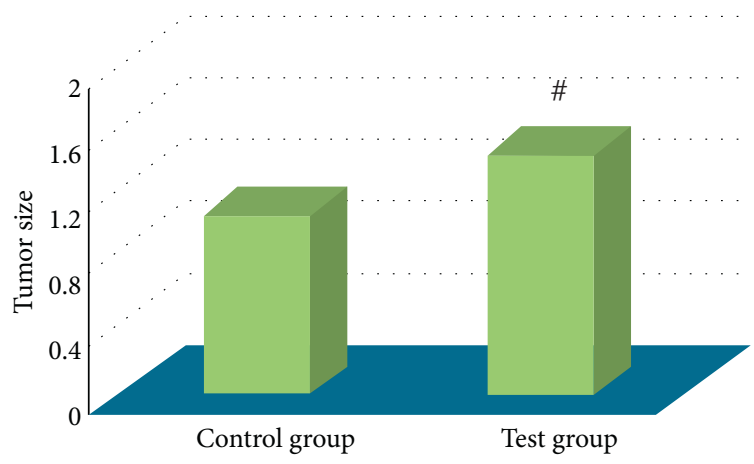

Figure 7: Comparison of tumor size between two groups of patients. Note: \# indicates remarkable difference in contrast to that of control group, $P<0.05$.

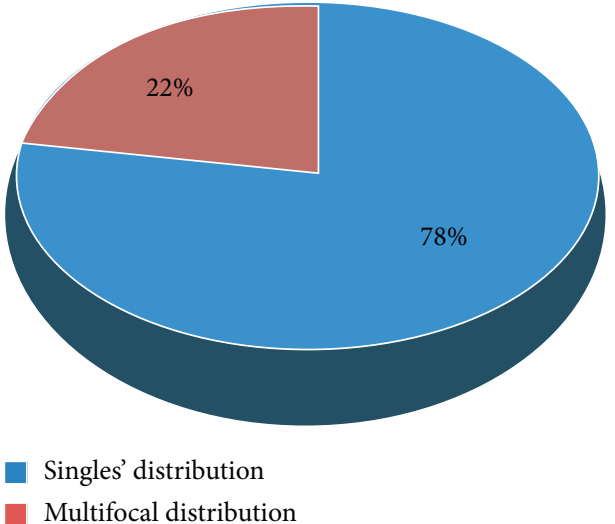

(a)
Multifocal distribution

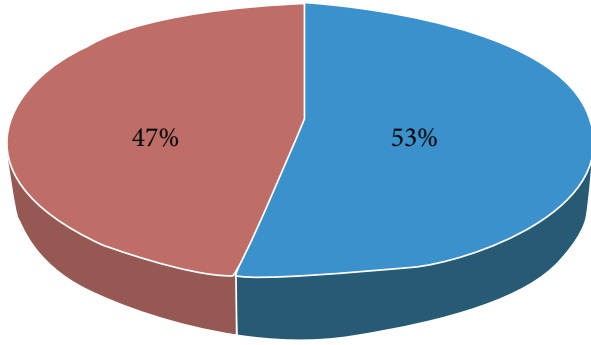

Singles' distribution

Multifocal distribution

(b)

Figure 8: The distribution of the number of lesions in the two groups of patients. (a) Test group; (b) control group.

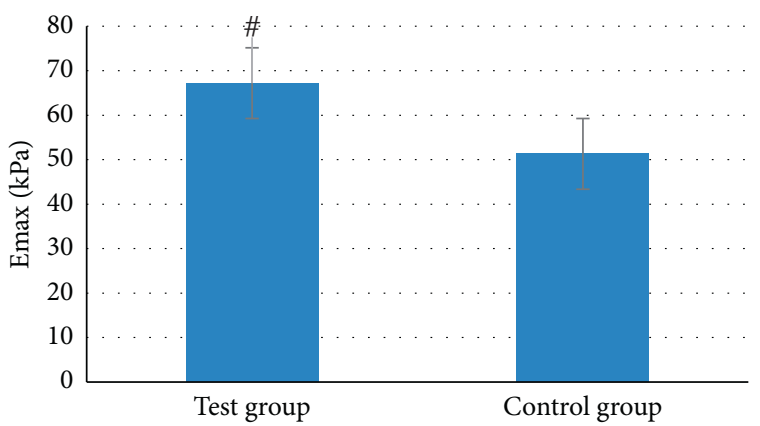

Figure 9: Comparison of $E_{\max }$ values between the two groups of patients. Note: \# indicates remarkable difference in contrast to that of control group, $P<0.05$.

and lymph node metastasis, making the implementation of surgical resection very difficult $[12,13]$. As an emerging technology, real-time SWE is widely used in assisted medical imaging diagnosis and analysis of various cancers [14, 15]. At present, the preoperative diagnosis of thyroid cancer using SWE is relatively common [16, 17]. However, the traditional method of using the physician's subjective judgment will easily lead to misdiagnosed cases. Therefore, how to accurately locate the tumor lesions and lymph nodes still needs further research.

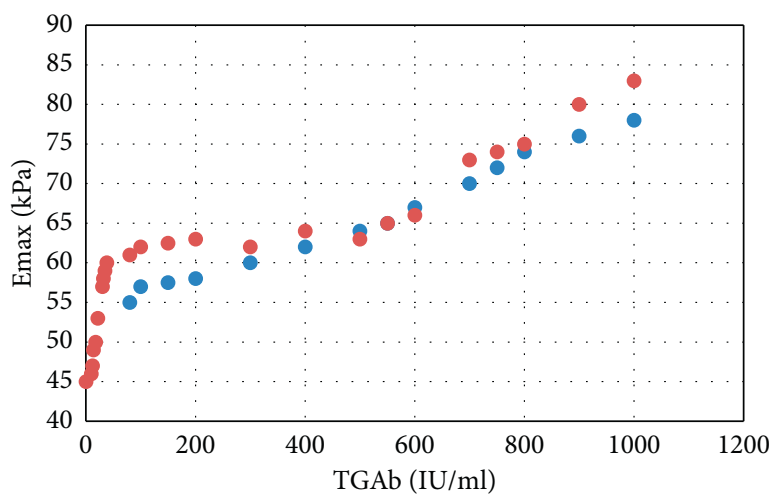

FIgure 10: Correlation distribution of $E_{\max }$ value and TGAb.

In this work, the real-time SWE based on the hybrid displacement estimation algorithm was adopted to automate the image segmentation to improve the image processing effect, by which the macroscopic and microscopic imaging omics characteristics were obtained. TGAb detection was used as an auxiliary diagnosis method for DTC [18]. ROC curve was used to analyze the sensitivity, specificity, and AUC of joint detection of real-time SWE combined with TGAb to detect the malignant degree of thyroid cancer. It was found that, in 102 patients with thyroid cancer, the 


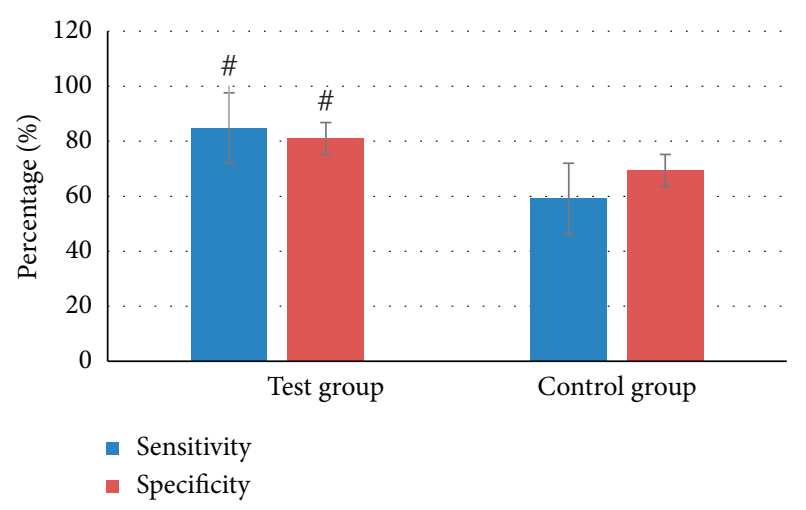

Figure 11: Comparison of the sensitivity and specificity of thyroid cancer detection between the two groups of patients. Note: \# indicates remarkable difference in contrast to that of control group, $P<0.05$.

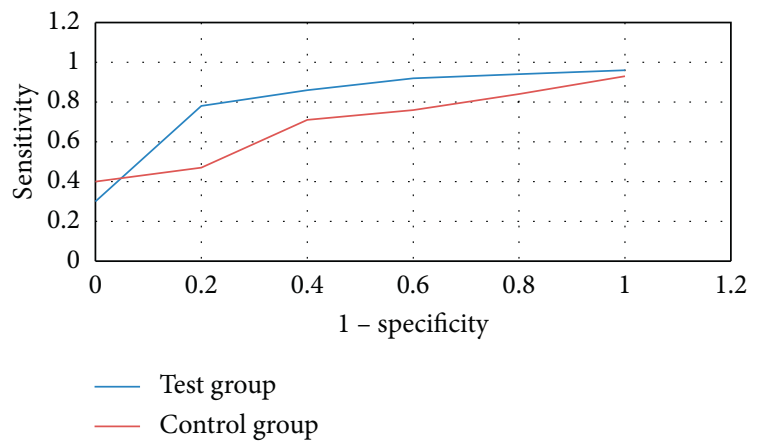

FIGURE 12: Comparison of AUC of thyroid cancer detection between two groups of patients.

preoperative TSH, TGAb, and TPOAb of experimental group were greater than those of the control group $(P<0.05)$. The preoperative tumors of experimental group were multifocal, and the tumor size was larger than that of TGAb-positive patients $(P<0.05)$. Such results were similar to the results of Patel et al. [19], indicating that TGAb levels directly affected the tumor size. $E_{\max }$ of Young's modulus of experimental group was greater than that of the control group $(P<0.05)$. There was a very significant positive correlation between the TGAb level and $E_{\max }$ of Young's modulus $(P<0.001)$. Moreover, the sensitivity, specificity, and AUC of the joint detection of real-time SWE $E_{\max }$ and TGAb for the malignant degree of thyroid cancer were significantly greater than those of the single real-time SWE and TGAb, and the difference was substantial $(P<0.05)$. The joint detection of SWE $E_{\max }$ and TGAb had high prediction accuracy and AUC and can be used as an improved model for predicting thyroid cancer. In short, joint detection of real-time SWE based on hybrid displacement estimation algorithm combined with TGAb had high sensitivity, specificity, and AUC for the diagnosis of DTC, which was suitable for clinical application.

\section{Conclusion}

In this study, real-time SWE was optimized based on mathematical imaging algorithms, combined with TGAb, and applied to the clinical imaging diagnosis and analysis of
102 cases of DTC. It was found that the joint detection of real-time SWE based on the optimization of the imaging algorithm combined with TGAb showed high sensitivity, specificity, and AUC for the diagnosis and prediction of DTC. However, the selection of patient samples in this study is small and the source is single, and the different manifestations are not discussed in detail, which makes it impossible to verify the influence of these characteristics on the accuracy of diagnosis. In the future, it should be considered to increase the sample size of patients with thyroid cancer and further adopt a multicenter collaborative analysis method for research. In conclusion, the results provide a good theoretical basis for the optimized real-time SWE algorithm combined with TGAb in the clinical diagnosis of DTC.

\section{Data Availability}

No data were used to support this study.

\section{Conflicts of Interest}

The authors declare that they have no conflicts of interest.

\section{Acknowledgments}

This work was supported by Qiqihar Science and Technology Bureau Joint Guidance (fund no. LHYD-202039).

\section{References}

[1] P. Pasyar, S. Masjoodi, Z. Montazeriani, and B. Makkiabadi, "A digital viscoelastic liver phantom for investigation of elastographic measurements," Computers in Biology and Medicine, vol. 127, Article ID 104078, 2020.

[2] W.-B. Zhang, J.-J. Li, X.-Y. Chen et al., "SWE combined with ACR TI-RADS categories for malignancy risk stratification of thyroid nodules with indeterminate FNA cytology," Clinical Hemorheology and Microcirculation, vol. 76, no. 3, pp. 381390, 2020.

[3] X. Hu, Y. Liu, and L. Qian, "Diagnostic potential of real-time elastography (RTE) and shear wave elastography (SWE) to differentiate benign and malignant thyroid nodules," Medicine, vol. 96, no. 43, Article ID e8282, 2017.

[4] V. Cantisani, E. David, H. Grazhdani et al., "Prospective evaluation of semiquantitative strain ratio and quantitative $2 \mathrm{D}$ ultrasound shear wave elastography (SWE) in association with TIRADS classification for thyroid nodule characterization," European Journal of Ultrasound, vol. 40, no. 4, pp. 495-503, 2019.

[5] H. Monpeyssen, J. Tramalloni, S. Poirée, O. Hélénon, and J.-M. Correas, "Elastography of the thyroid," Diagnostic and Interventional Imaging, vol. 94, no. 5, pp. 535-544, 2013.

[6] G. Azizi, K. Faust, M. L. Mayo, J. Farrell, and C. Malchoff, "Diagnosis of thyroid nodule with new ultrasound imaging modalities," VideoEndocrinology, vol. 7, no. 1, 2020.

[7] S. G. A. de Meer, W. M. C. M. Vorselaars, J. W. Kist et al., "Follow-up of patients with thyroglobulin-antibodies: rising $\mathrm{Tg}-\mathrm{Ab}$ trend is a risk factor for recurrence of differentiated thyroid cancer," Endocrine Research, vol. 42, no. 4, pp. 302-310, 2017. 
[8] S.-J. Kim, S.-W. Lee, K. Pak, and S.-R. Shim, "Diagnostic performance of PET in thyroid cancer with elevated anti-Tg Ab," Endocrine-Related Cancer, vol. 25, no. 6, pp. 643-652, 2018.

[9] U. Mousa, A. S. Yikilmaz, and A. Nar, "Stimulated thyroglobulin values above $5.6 \mathrm{ng} / \mathrm{ml}$ before radioactive iodine ablation treatment following levothyroxine withdrawal is associated with a 2.38 -fold risk of relapse in $\mathrm{Tg}$-ab negative subjects with differentiated thyroid cancer," Clinical and Translational Oncology, vol. 19, no. 8, pp. 1028-1034, 2017.

[10] M. Yéléhé-Okouma, C. Malaplate, N. Petitpain et al., "Immunoglobulin preparations can mislead clinical decisionmaking in follow-up of differentiated thyroid cancer," Endocrine Practice, vol. 26, no. 9, pp. 1031-1038, 2020.

[11] S. R. Zakavi, N. Ayati, S. Zare et al., "Prognostic value and optimal threshold of first thyroglobulin in low/intermediate risk DTC," The Quarterly Journal of Nuclear Medicine and Molecular Imaging, vol. 65, no. 1, pp. 64-71, 2021.

[12] E. Giannoula, C. Melidis, N. Papadopoulos, P. Bamidis, V. Raftopoulos, and I. Iakovou, "Dynamic risk stratification for predicting treatment response in differentiated thyroid cancer," Journal of Clinical Medicine, vol. 9, no. 9, Article ID 2708, 2020.

[13] B. İ. Aydoğan, A. B. B. Mutlu, S. Yüksel et al., "The Association of histologically proven chronic lymphocytic thyroiditis with clinicopathological features, lymph node metastasis, and recurrence rates of differentiated thyroid cancer," Endocrine Pathology, vol. 32, no. 2, pp. 280-287, 2020.

[14] M. H. Yoo, H. J. Kim, I. H. Choi et al., "Shear wave elasticity by tracing total nodule showed high reproducibility and concordance with fibrosis in thyroid cancer," BMC Cancer, vol. 20 , no. 1, p. 118, 2020.

[15] U. Mousa, A. S. Yikilmaz, and A. Nar, "Erratum to: stimulated thyroglobulin values above $5.6 \mathrm{ng} / \mathrm{ml}$ before radioactive iodine ablation treatment following levothyroxine withdrawal is associated with a 2.38 -fold risk of relapse in $\mathrm{Tg}$-ab negative subjects with differentiated thyroid cancer," Clinical and Translational Oncology, vol. 19, no. 11, p. 1421, 2017.

[16] S. Hwang, D. Y. Shin, E. K. Kim et al., "Focal lymphocytic thyroiditis nodules share the features of papillary thyroid cancer on ultrasound," Yonsei Medical Journal, vol. 56, no. 5, pp. 1338-1344, 2015.

[17] S. A. Rakib, S. B. Sharif, A. Nahar, and S. M. Alam, "Prediction of thyroid malignancies by thyroid auto antibodies," Mymensingh Medical Journal: Mie Medical Journal, vol. 27, no. 3, pp. 585-595, 2018.

[18] S. Ha, S. W. Oh, Y. K. Kim et al., "Clinical outcome of remnant thyroid ablation with low dose radioiodine in Korean patients with low to intermediate-risk thyroid cancer," Journal of Korean Medical Science, vol. 30, no. 7, pp. 876-881, 2015.

[19] A. Patel, V. Shostrom, K. Treude, W. Lydiatt, R. Smith, and W. Goldner, "Serum thyroglobulin: preoperative levels and factors affecting postoperative optimal timing following total thyroidectomy," International Journal of Endocrinology, vol. 2019, Article ID 1384651, 8 pages, 2019. 